\title{
Spina bifida in fetus is associated with an altered pattern of DNA methylation in placenta
}

\author{
Xiaojuan Zhang ${ }^{1,2,6}$, Lijun Pei ${ }^{3,6}$, Runting $\mathrm{Li}^{1,2}$, Wei Zhang ${ }^{4,5}$, Hua Yang1, Yongchao $\mathrm{Li}^{1,5}$, Yu Guo ${ }^{1,5}$, \\ Pingping Tan ${ }^{1}$, Jingdong $\mathrm{J} \mathrm{Han}^{4,5}$, Xiaoying Zheng ${ }^{3}$ and Runlin $\mathrm{Z} \mathrm{Ma}^{1,2,5}$
}

Failure in closure of neural tube leads to neural tube defects (NTDs), which are among the most common symptoms of human birth defects. Although epigenetic status in placenta is linked to fetal development, the mechanism behind this remains unknown. Because of the importance of DNA methylation in gene function, we set to explore whether or not DNA methylation in human placenta is also linked to fetal NTDs. Here we show for the first time that alteration of DNA methylation in placenta is closely associated with the phenotypes of fetal spina bifida (Sb). We found that patterns of DNA methylation for genes in neurological system process were differentially altered in the Sb placenta. In particular, the transcription regulatory regions of TRIM26 and GANS were kept at the hypomethylation status in Sb placenta alone. Accordingly, the protein levels of TRIM26 and GNAS were significantly elevated only in the Sb placenta but not in the Sb-affected fetuses. In cellular model of CHO cells deficient in Dihydrofolate reductase and treated with 5-aza-2'-deoxycytidine, the protein levels of GNAS and TRIM26 were significantly higher than those in normal control cells. These findings suggested that epigenetic status of genes in placenta have profound impacts on the development of NTDs.

Journal of Human Genetics (2015) 60, 605-611; doi:10.1038/jhg.2015.80; published online 16 July 2015

\section{INTRODUCTION}

A fetal neural tube is an embryonic precursor of the spinal cord and brain, budding from the neural plate and undergoing a series of events of shaping and folding during the early fetal development. Failure in initial fusion and closure results in neural tube defects (NTDs), which are among the most common forms of human birth defects. ${ }^{1}$ Spina bifida (Sb) and anencephaly are the most common NTDs, and each year $\sim 300000$ or more has been identified in newborns worldwide. ${ }^{2}$ There are geographic variations in the occurrence rate of $\mathrm{Sb}$ and anencephaly and the highest incidence in Northern China. ${ }^{2}$ A survey during the years 2003-2004 showed that with 199.38 per 10000 births recorded in Shanxi province in Northern China. ${ }^{3}$ Despite more and more studies conducted worldwide about NTDs etiology, the molecular mechanisms are still poorly understood.

Placenta acts as a controller of intrauterine environmental conditions and fetal programming. ${ }^{4}$ Its functions are influenced by maternal exposures including nutrition, stress and psychology during pregnancy, ${ }^{5}$ which was often presented by epigenetic changes. Recent studies showed that DNA methylation alterations in placenta are linked to aberrant fetal growth and development. ${ }^{6,7}$ However whether placental DNA methylation alteration attributes to fetal $\mathrm{Sb}$ is not clear.

In this study we investigated the placental DNA methylation patterns of normal and Sb samples and found that it is significantly associated with $\mathrm{Sb}$ phenotype. Further data analysis showed that there are more than 20 differential genes involved in neurological system process and the status of DNA methylation in tripartite motifcontaining 26 (TRIM26) and guanine nucleotide-binding protein Gs(GNAS) exactly cause the alteration of their protein expression. The findings suggested that epigenetic alteration of placental genes may be an important factor attributing to fetal Sb.

\section{MATERIALS AND METHODS}

Sample collection

All the samples of human placenta used in this study were obtained from clinic hospitals in Shanxi Province (China). Ethics approval for the study was obtained through the human ethnic committees of the Capital Institute of Pediatrics and the Institute of Genetics and Developmental Biology, Chinese Academy of Sciences. Sample information collected from each subject included gestational weeks at delivery, newborn's gender and fetal symptom. Placental

\footnotetext{
${ }^{1}$ State Key Laboratory for Molecular Developmental Biology, Institute of Genetics and Developmental Biology, Chinese Academy of Sciences, Beijing, China; ${ }^{2}$ Laboratory of Molecular Biology, Zhengzhou Normal University, Zhengzhou, China; ${ }^{3}$ WHO Collaborating Center for Research in Reproductive Health and Population Science, Institute of Population Research, Peking University, Beijing, China; ${ }^{4}$ Chinese Academy of Sciences Key Laboratory of Computational Biology, CAS-MPG Partner Institute for Computational Biology, Shanghai Institutes for Biological Sciences, Chinese Academy of Sciences, Shanghai, China and ${ }^{5}$ Graduate University of the Chinese Academy of Sciences, University of Chinese Academy of Sciences, Beijing, China

${ }^{6}$ These authors contributed equally to this work.

Correspondence: Professor X Zheng, WHO Collaborating Center for Research in Reproductive Health and Population Science, Institute of Population Research, Peking University, Beijing 100871, China.

E-mail: xzheng@pku.edu.cn

or Professor RZ Ma, State Key Laboratory for Molecular Developmental Biology, Institute of Genetics and Developmental Biology, Chinese Academy of Sciences, S2-310 West Beichen Road, Chaoyang District, Beijing 100101, China.

E-mail: rlma@genetics.ac.cn
}

Received 11 December 2014; revised 10 June 2015; accepted 12 June 2015; published online 16 July 2015 
specimens were collected by OB/GYN (obstetrics/gynecology) delivery doctors and the samples were stored at $-80^{\circ} \mathrm{C}$ until use. Because most of babies born with the syndrome of NTDs were aborted incompletely, it was extremely hard to match the gestation age between $\mathrm{Sb}$ and normal placental samples. After initial screening for the quality of total RNA isolated from each placenta sample, a total of 16 biological replicates (eight $\mathrm{Sb}$ and eight normal controls) were chosen for further studies. Table 1 describes the characteristics of all samples.

\section{DNA Methylation Microarray assay}

Genomic DNA from both $\mathrm{Sb}$ and control placenta were extracted using EasyPure Genomic DNA Kit (TransGen Biotech, Beijing, China) following manufacturer's protocols. Purified DNA samples were checked by $1 \%$ agarose elelectrophoresis and quantified with the NanoDrop ND1000 spectrophotometer (Thermo Scientific, Wilmington, DE, USA). Qualified DNA samples were selected for the subsequent analysis of DNA methylation. Briefly, $1 \mu \mathrm{g}$ of each placental DNA was bisulfite modified using EZ DNA Methylation Kit D5008 (Zymo Research, Irvine, CA, USA) according to the manufacturer's recommendations for the Illumina Infinium Assay (Illumina, San Diego, CA, USA). All the bisulfite-converted DNA samples were hybridized on Infinium Human Methylation450 BeadChip following standardized protocols and the signals were scanned using the Illumina HiScan SQ scanner (Illumina). The methylation score for each CpG was represented as a $\beta$ value according to the fluorescent intensity ratio. ${ }^{8}$

\section{Data analysis and statistical treatment}

Initial data was analyzed following that procedure showed in Supplementary figure S1. To find the differential methylation signal of each group, we used RankProd package (The Salk Institute, La Jolla, CA, USA). From the results showed in Supplementary table S1 we decided to choose $P$-value $=0.01$ and $\Delta \mathrm{FC}=0.2$ to be cutoff. Clustering analysis (Clusters 1 and 2) were done using MeV2.0 (Open Source Initiative, Palo Alto, CA, USA). The data of Cluster 2 was from the site located in transcription start sites (TSS) region. Based on the sites in Cluster 2 we removed the single-nucleotide polymorphism-containing probes and did gene ontology (GO) enrichment analysis. GO terms were calculated as described by Xia et al. ${ }^{9}$ Basically, Fisher's exact test was used to estimate the significance levels of gene set enrichment and the BenjaminiHochberg corrected false discovery rate $\leqslant 0.05$ was used as the cutoff.

\section{Bisulphite PCR sequencing}

To test the methylation pattern of TRIM26 and GNAS in placenta, the tested region and ite flanking sequences were retrieved for primer design for bidulfite genomic sequencing. The primers for TRIM26 gene follow as: TRIM26-F 5'- ATTAGGGTTYGAGGGYGTTTTG-3' and TRIM26-R 5'-CAAAACATA

Table 1 Information of the samples involved in the study

\begin{tabular}{lccc}
\hline Sample & Fetal gender & Gestational age (week) & Fetal symptom \\
\hline Ctrl11 & Male & 40 & Normal \\
Ctrl12 & Male & 40 & Normal \\
Ctrl13 & Male & 40 & Normal \\
Ctrl14 & Male & 40 & Normal \\
Ctrl21 & Female & 40 & Normal \\
Ctrl22 & Female & 40 & Normal \\
Ctrl23 & Female & 40 & Normal \\
Ctrl24 & Female & 40 & Normal \\
Sb11 & Male & 40 & $\mathrm{Sb}$ \\
Sb12 & Male & 41 & $\mathrm{Sb}$ \\
Sb13 & Male & 30 & $\mathrm{Sb}$ \\
Sb14 & Male & 31 & $\mathrm{Sb}$ \\
Sb21 & Female & 39 & $\mathrm{Sb}$ \\
Sb22 & Female & 30 & $\mathrm{Sb}$ \\
Sb23 & Female & 22 & $\mathrm{Sb}$ \\
Sb24 & Female & 27 & $\mathrm{Sb}$ \\
\hline Abbrition & & &
\end{tabular}

Abbreviations: Ctrl, control; Sb, spina bifida.
ACCCTACRACTAAACC-3'. The primers for GNAS genes is GNAS-F 5'-TTTGTTGTTTTYGAGTGTTTTTGGG-3' and GNAS-R 5' CCACACTACA CAACRATAATAATATC-3'. Genomic DNA from both three Sbs and three control placentas were was bisulfite modified using EZ DNA Methylation Kit D5008 (Zymo Research) according to the manufacturer's recommendations. The bisulfite-converted DNA samples were used for PCR amplification. The amplified products were gel-purified and then subjected to TA-cloning. Ten clones for each cases were selected DNA sequencing.

\section{Western blot analysis}

To verify the effects of differential DNA methylation between $\mathrm{Sb}$ and normal control placenta, total protein samples were isolated for each individual sample using tissue lysis buffer (20 mm Tris-Cl [pH8.0], 0.2 M NaCl, 0.5\% NP40, $1 \mathrm{~mm}$ EDTA, $0.1 \%$ SDS and $1 \times$ protease inhibitor mixture). The protein concentration for each sample was determined by a Bicinchoninic acid (BCA) assay. Equal amounts of total proteins were resolved on a 10\% SDS-polyacrylamide gel electrophoresis) gels and transferred onto a nitrocellulose membrane. The membranes were blocked for $1 \mathrm{~h}$ in Tris-buffered saline containing $0.2 \%$ Tween 20 and $5 \%(\mathrm{w} / \mathrm{v})$ nonfat milk, and subsequently probed $2 \mathrm{~h}$ at room temperature with rabbit polyclonal anti-GNAS (ab58916, 1:1000, Abcam, Cambridge, UK), mouse monoclonal anti-TRIM26 (ab89290, 1:1000, Abcam), and rabbit polyclonal anti-GAPDH (SC-25778, 1:1000, Santa Cruz Biotechnology, Dallas, TX, USA). The membranes were then incubated for $1 \mathrm{~h}$ at room temperature with secondary horseradish peroxidase-conjugated anti-rabbit (1:3000, ZSGB-BIO, Beijing, China) or anti-mouse IgG $(\mathrm{H}+\mathrm{L})$ (1:3000, ZSGB-BIO). The blots were developed in Super Signal West Durachemiluminescence (Thermo Scientific, Waltham, MA, USA) according to the manufacturer's instruction.

\section{Cell culture and treatment}

To test whether or not the protein levels of GNAS and TRIM26 could be affected by the methylation level and the deficiency of folic acid. 5-aza-2'deoxycytidine, a DNA methytransferase inhibitor, wad used to treat cells for testing the effect of DNA methylation status on the protein levels of GNAS and TRIM26. Cells were treated in 5-aza-2'-deoxycytidine $(10 \mu \mathrm{M})$ for $72 \mathrm{~h}$ and then harvested for western blot assay. The $\mathrm{CHO}$ and $\mathrm{CHO} / \mathrm{dhfr}$ - cells were obtained commercially (ATCC, Rockefeller, MD, USA) and were grown in DMEM/F12 media according to the supplier's recommendation. Cells at the identical culture condition were harvested in lysis buffer containing $20 \mathrm{~mm}$ Tris$\mathrm{Cl}$ [pH8.0], $0.2 \mathrm{M} \mathrm{NaCl}, 0.5 \% \mathrm{NP} 40,1 \mathrm{~mm}$ EDTA and $1 \times$ protease inhibitor mixture for western blot analysis.

\section{RESULTS}

Placental DNA methylation patterns are significantly associated with spina bifida

To reveal the placental DNA methylation pattern of Sb, Infinium Human Methylation450 BeadChip was used. Table 1 describes the characteristics of all samples involved in this study. Eight samples in each group consist of four males and four females for testing the effect of fetal sex on DNA methylation profile. Initial data was analyzed following that procedure showed in Methods. According to Supplementary table S1 and the cutoff, the total amount of differential sites is 3839 , which are used to followed-by clustering analysis. The upper panel in Figure 1 showed the clustered analysis result. In 3839 sites, there are 914 in hypermethylation status in Sb samples corresponding to 163 genes, and additional 2925 probes are hypomethylation sites in $\mathrm{Sb}$ samples corresponding to 810 genes. So in $\mathrm{Sb}$ the number of hypomethylation sites is far more than that of hypermethylation sites, which suggests that a large number of genes are activated, transcribed and even translated in Sb placenta.

Based on the above analysis, we obtained the location information of each probe and then picked the sites located in TSS regions. And those sites were deleted which are related to fetal sex and those unmatched probes (DNA methylation variation in conflict in the same gene). The panel in Figure 1 presents the clustering analysis result. 


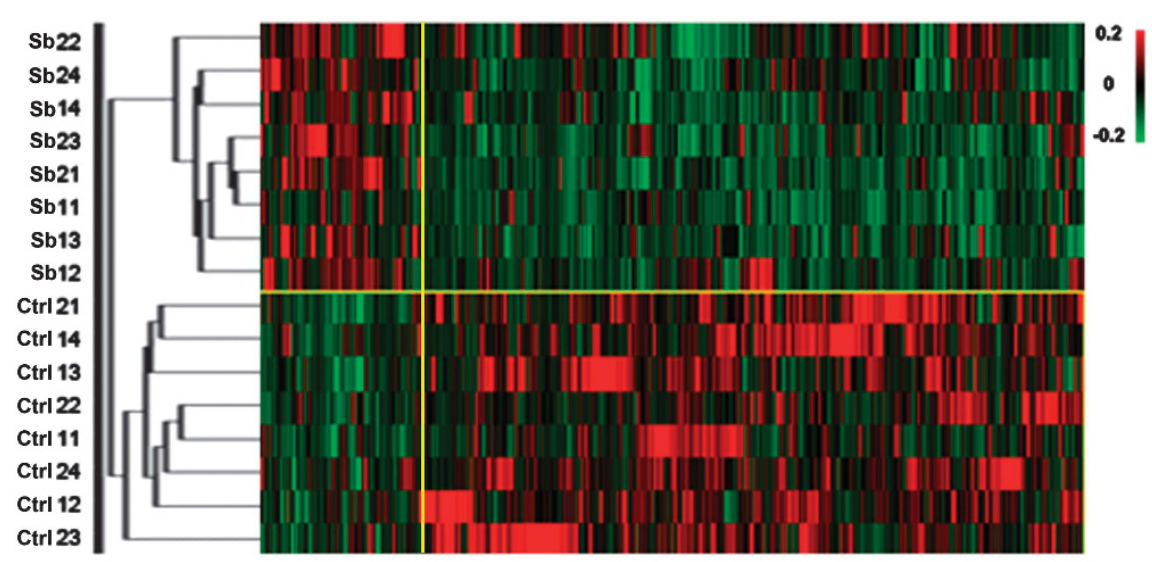

Figure 1 Clustering analysis of differentially methylated sites between Sb and control samples in TSS region and after removing sex and age-related genes. Red/green gradient represents standardized level of hypermethylation/hypomethylation in Sb compared to controls. Cutoff: $P<0.01, \Delta \mathrm{FC}>0.2, \mathrm{Sex} P>0.05$ and Age R2 $<0.16$.

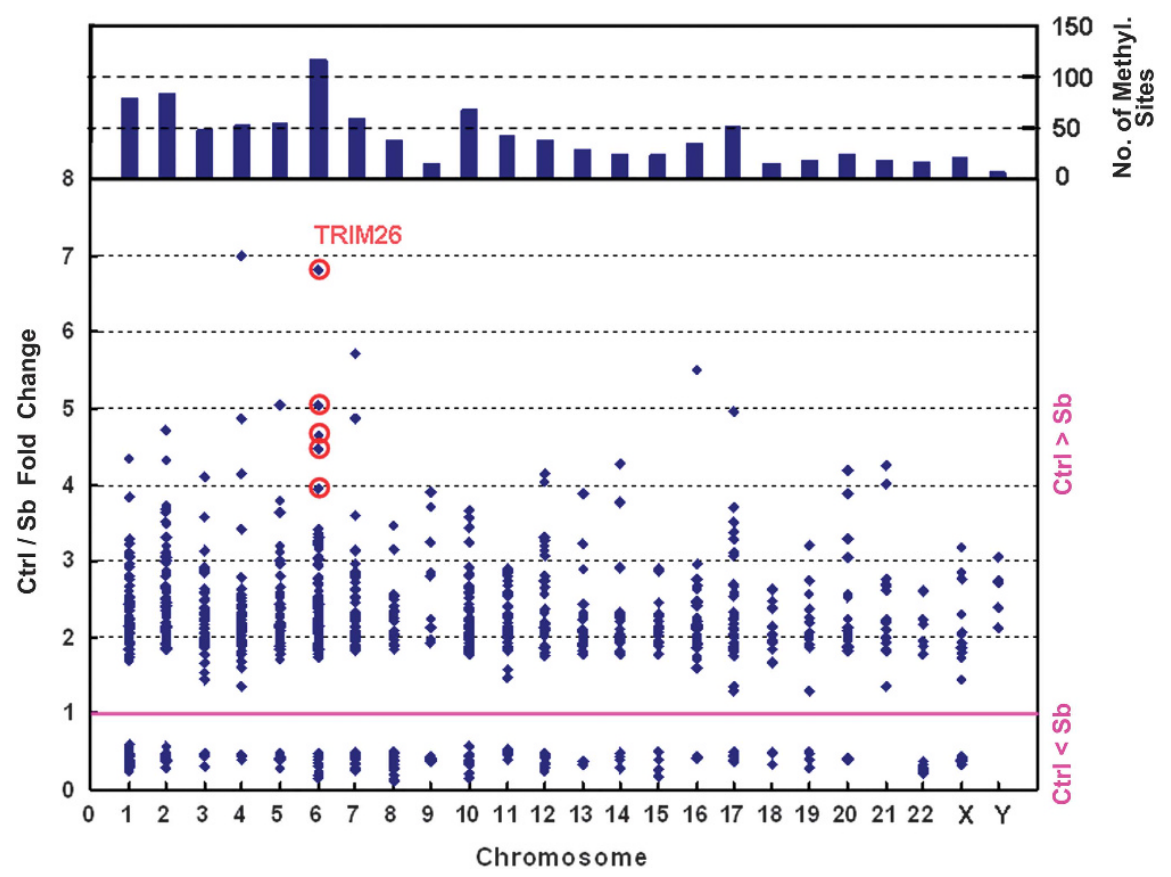

Figure 2 Chromosomal distributions of DNA methylation differential sites. Ctrl means control group and Sb means spinda bifida group. Ctrl<Sb means that DNA methylation level in the group of $\mathrm{Sb}$ is significantly higher than that of control. Ctrl $>\mathrm{Sb}$ shows that the methylation level of Sb group is dramatically lower than that of control $(P<0.01)$.

Twice clustering analysis suggests that placental DNA methylation patterns are dramatically changed in Sb.

Next we analyzed the distribution of these DNA methylation sites in chromosomes. The upper panel of Figure 2 presents the total number of sites in each chromosome. From this panel we found that the amount of differential sites in each chromosome is not correlated to the size of the chromosome, which hints that DNA methylation patterns are chromosomal specific. In chromosome 6 there are 119 differential sites which is a peak in all chromosomes. Interestingly all first five maximum of $\mathrm{Ctrl} / \mathrm{Sb}$ fold change point to the same gene, TRIM26. ('Ctrl' means control; 'Sb' means spina bifida.) In addition in chromosome 21 and Y, there is no differential hypermethylation locus in $\mathrm{Sb}$ at this level. The findings suggest that in the placenta selected DNA loci are demethylated or methylated.
The alteration of DNA methylation profile in some genes related to neurological system process in placentas of spina bifida

We subsequently performed GO analysis to explore the correlation between DNA methylation and gene function. Firstly we removed the single-nucleotide polymorphism-containing probes. The selected cutoff is $P<0.05$ and Fold $(\mathrm{Ctrl} / \mathrm{Sb})>4$ or Fold $(\mathrm{Ctrl} / \mathrm{Sb})<0.25$. Fold $(\mathrm{Ctrl} / \mathrm{Sb})>4$ means that in Sb DNA methylation signal value is more than fourfold lower than that in control (Ctrl), and vice versa. These genes are classified into five categories in Table 2. We found that some genes are directly involved in regulation of neurological system development and transport neurotransmitters.

Table 3 presented the DNA methylation information of every gene related to neurogenesis. Apparently in Sb samples those loci involved in neurogenesis are in condition of hypomethylation $(\mathrm{Ctrl}>\mathrm{Sb})$, and 
Table 2 The GO annotation of the genes found with differential DNA methylation in TSS region; Cutoff: $P<0.05 ;$ Fold (Ctrl/Sb) $>4$ or Fold (Ctrl/Sb) $<0.25$

\begin{tabular}{|c|c|c|c|c|}
\hline Types & GO Terms & P-value & $\begin{array}{l}\text { Fold } \\
\text { (Ctrl/Sb) }\end{array}$ & Genes \\
\hline \multirow[t]{18}{*}{$\begin{array}{l}\text { 1. Neurological system } \\
\text { development and process. }\end{array}$} & $\begin{array}{l}\text { G0:0007406 I negative regulation of neuroblast } \\
\text { proliferation }\end{array}$ & 0.0091 & 52.98 & $V A X 1 ; B D N F$ \\
\hline & GO:0014032 I neural crest cell development & 0.0194 & 31.79 & Sox9 \\
\hline & G0:0021549I cerebellum development & 0.0227 & 28.90 & $L M X 1 A ; L H X 1$ \\
\hline & G0:0035295I tube development & 0.0002 & 24.08 & $G D N F ; B D N F ; B M P 7 ; L H X 1$ \\
\hline & G0:0042596 I fear response & 0.0434 & 17.66 & $B D N F$ \\
\hline & G0:0050905I neuromuscular process & 0.0194 & 12.55 & KCNMA1; HMX3 \\
\hline & GO:0001764 I neuron migration & 0.0056 & 12.23 & FEZF1; VAX1 \\
\hline & $\begin{array}{l}\text { GO:0050768 I negative regulation of } \\
\text { neurogenesis }\end{array}$ & 0.0276 & 10.60 & $V A X 1 ; B D N F ; B M P 7$ \\
\hline & G0:0030182 I neuron differentiation & 0.0003 & 10.30 & PTPRR; KCNMA1; BDNF; LMXIA; LHXI; IRX5 \\
\hline & GO:0007411 Iaxon guidance & 0.0049 & 8.73 & FEZF1; VAX1; BDNF; BMP7; LMX1A \\
\hline & GO:0007423 I sensory organ development & 0.0434 & 8.37 & $V A X 1$ \\
\hline & G0:0007420 I brain development & 0.0276 & 6.76 & VAX1; HMX3 \\
\hline & GO:0050767 | regulation of neurogenesis & 0.0091 & 5.81 & $\angle B X 1 ; V A X 1 ; B D N F ; B M P 7 ; \angle M X 1 A$ \\
\hline & GO:0007399 I nervous system development & 0.0013 & 4.77 & FEZF1; LBX1; PTPRR; PLXNA4; FAM5B; HMX3; SIM2; LHX1 \\
\hline & G0:0050877 I neurological system process & 0.0193 & 4.52 & GNAS; PENK; GALR2 \\
\hline & G0:0006836 I neurotransmitter transport & 0.0389 & 0.04 & $S V 2 C ; R I M S 1$ \\
\hline & GO:0042165 I neurotransmitter binding & 0.0389 & 0.04 & NMUR1; GRIN3A \\
\hline & GO:0045202 I synapse & 0.0389 & 0.09 & SV2C; RIMS1; GRIN3A \\
\hline \multirow{17}{*}{$\begin{array}{l}\text { 2. Cell development and } \\
\text { differentiation }\end{array}$} & G0:0048762 I mesenchymal cell differentiation & 0.0064 & 63.57 & $B M P 7$ \\
\hline & GO:0001657/ ureteric bud development & 0.0000 & 39.73 & GDNF; BDNF; BMP7; LHX1 \\
\hline & G0:0021549I cerebellum development & 0.0227 & 28.90 & $L M X 1 A ; L H X 1$ \\
\hline & G0:0014031 I mesenchymal cell development & 0.0248 & 26.49 & sox9 \\
\hline & G0:0042490 I mechanoreceptor differentiation & 0.0336 & 21.19 & KCNMA1; BDNF \\
\hline & G0:0001704 I formation of primary germ layer & 0.0013 & 19.87 & BMP7; LHX1; WNT3 \\
\hline & G0:0001707 I mesoderm formation & 0.0092 & 17.66 & BMP7; WNT3 \\
\hline & G0:0009880 I embryonic pattern specification & 0.0125 & 15.38 & BMP7; SIM2; LHX1 \\
\hline & GO:0051216I cartilage development & 0.0229 & 11.63 & GNAS; BMP7; BMP3 \\
\hline & G0:0001503। ossification & 0.0092 & 10.09 & GNAS; FOXC1; BMP7; BMP3 \\
\hline & $\begin{array}{l}\text { GO:0010721 I negative regulation of cell } \\
\text { development }\end{array}$ & 0.0317 & 9.73 & $V A X 1 ; B D N F ; B M P 7$ \\
\hline & GO:0060284 | regulation of cell development & 0.0181 & 4.87 & $L B X 1 ; V A X 1 ; B D N F ; B M P 7 ; L M X 1 A$ \\
\hline & G0:0003002 I regionalization & 0.0353 & 4.73 & PCDH8; LHX1; WNT3 \\
\hline & GO:0048468I cell development & 0.0231 & 4.50 & BMP7; EN2; HOXD9; IRX5; SOX9 \\
\hline & GO:0007389I pattern specification process & 0.0126 & 4.47 & BMP7; PCDH8; SIM2; LHX1; WNT3 \\
\hline & $\begin{array}{l}\text { GO:0032989 I cellular component } \\
\text { morphogenesis }\end{array}$ & 0.0429 & 4.46 & NKX2-8; BMP7; SLITRK5; SOX9 \\
\hline & G0:0016477Icell migration & 0.0336 & 4.01 & FEZF1; VAX1; GDNF \\
\hline \multirow[t]{2}{*}{ 3. Transcription regulation } & $\begin{array}{l}\text { G0:0003702 IRNA polymerase II transcription } \\
\text { factor activity }\end{array}$ & 0.0096 & 4.73 & NKX2-8; LHX1; MAF; SOX9 \\
\hline & G0:0043565Isequence-specific DNA binding & 0.0000 & 4.66 & $\begin{array}{l}\text { LBX1; DBX1; VAX1; EVX2; NKX2-8; GSC2; HMX3; } \\
\text { EN2; ESX1; LMX1A; LHX1; IRX5; MAF; SOX9 }\end{array}$ \\
\hline \multirow[t]{6}{*}{$\begin{array}{l}\text { 4. Cell signaling and } \\
\text { movement }\end{array}$} & $\begin{array}{l}\text { G0:0001837 I epithelial-to-mesenchymal } \\
\text { transition }\end{array}$ & 0.0415 & 18.70 & BMP7; SOX9 \\
\hline & G0:0008344 I adult locomotory behavior & 0.0317 & 9.73 & KCNMA1; GDNF \\
\hline & G0:0008083 I growth factor activity & 0.0317 & 4.94 & GDNF; BDNF; BMP7; BMP3; INHBB \\
\hline & G0:0048870 I cell motility & 0.0185 & 4.12 & FEZF1; VAX1; GDNF \\
\hline & GO:0006928I cellular component movement & 0.0021 & 4.07 & FEZF1; VAX1; GDNF; BDNF; BMP7; TPM1; LMX1A \\
\hline & GO:0007154 I cell communication & 0.0003 & 4.04 & $\begin{array}{l}\text { VIPR2; KCNIP1; KCNMA1; GDNF; SH3PXD2B; BDNF; BMP3; } \\
\text { PCDH8; LHX1; WNT3 }\end{array}$ \\
\hline \multirow[t]{3}{*}{$\begin{array}{l}\text { 5. Biosynthetic and } \\
\text { metabolic process }\end{array}$} & $\begin{array}{l}\text { GO:0008535 I respiratory chain complex IV } \\
\text { assembly }\end{array}$ & 0.0132 & 39.73 & $\operatorname{cox} 11 ; \operatorname{SCO} 2$ \\
\hline & G0:0006816I calcium ion transport & 0.0137 & 0.04 & NMUR1; GRIN3A \\
\hline & $\begin{array}{l}\text { GO:0015674Idi-; tri-valent inorganic cation } \\
\text { transport }\end{array}$ & 0.0172 & 0.05 & NMUR1; GRIN3A \\
\hline
\end{tabular}

Abbreviations: GO, gene ontology; TSS, transcription start sites. 
Table 3 DNA Methylation information of the genes about neurological system process

\begin{tabular}{|c|c|c|c|c|c|c|}
\hline Type & & Gene & Gene full name & $\begin{array}{c}\text { No. of sites } \\
\text { in gene }\end{array}$ & Location & $\begin{array}{l}\text { Avg. of Ctrl/Sb } \\
\text { fold change }\end{array}$ \\
\hline \multirow[t]{18}{*}{$\mathrm{Ctrl}>\mathrm{Sb}$} & 1 & TRIM26 & Tripartite motif-containing 26 & 20 & TSS1500 & 3.105 \\
\hline & 2 & $L H X 1$ & LIM homeobox 1 & 2 & TSS1500;Body & 2.753 \\
\hline & 3 & FEZF1 & FEZ family zinc finger 1 & 5 & 1stExon; TSS1500 & 2.492 \\
\hline & 4 & PLXNA4 & Plexin A4 & 2 & TSS1500;TSS1500; $5^{\prime}$ UTR & 2.407 \\
\hline & 5 & FAM5B & Family with sequence similarity 5 , member $B$ & 1 & TSS1500 & 2.276 \\
\hline & 6 & KCNMA1 & Potassium large conductance calcium-activated channel, subfamily M, a & 3 & 1stExon;5'UTR;TSS1500 & 2.233 \\
\hline & 7 & $H M X 3$ & H6 family homeobox 3 & 1 & TSS200 & 2.222 \\
\hline & 8 & $\operatorname{IRX5}$ & Iroquois homeobox 5 & 2 & TSS200;Body & 2.173 \\
\hline & 9 & $V A X 1$ & Ventral anterior homeobox 1 & 4 & 3'UTR;TSS1500;Body & 2.162 \\
\hline & 10 & GDNF & Glial cell derived neurotrophic factor & 1 & TSS200 & 2.141 \\
\hline & 11 & PTPRR & Protein tyrosine phosphatase, receptor type, $\mathrm{R}$ & 1 & TSS200 & 2.123 \\
\hline & 12 & SIM2 & Single-minded homolog 2 (Drosophila) & 2 & TSS1500; Body & 2.117 \\
\hline & 13 & $B D N F$ & Brain-derived neurotrophic factor & 1 & TSS200,TSS1500 & 2.107 \\
\hline & 14 & $L B X 1$ & Ladybird homeobox 1 & 1 & TSS200 & 2.098 \\
\hline & 15 & GNAS & GNAS complex locus & 4 & 3'UTR;TSS1500;Body & 2.086 \\
\hline & 16 & $L M X 1 A$ & LIM homeobox transcription factor 1 , alpha & 2 & TSS1500 & 2.006 \\
\hline & 17 & BMP7 & Bone morphogenetic protein 7 & 2 & TSS200,TSS1500 & 1.911 \\
\hline & 18 & soxg & SRY (sex determining region Y)-box 9 & 1 & TSS1500 & 1.872 \\
\hline \multirow[t]{4}{*}{$\mathrm{Ctrl}<\mathrm{Sb}$} & 19 & GRIN3A & Glutamate receptor, ionotropic, $\mathrm{N}$-methyl-D-aspartate 3A & 1 & TSS200 & 0.436 \\
\hline & 20 & SV2C & Synaptic vesicle glycoprotein $2 \mathrm{C}$ & 2 & TSS200,TSS1500 & 0.415 \\
\hline & 21 & NMUR1 & Neuromedin U receptor 1 & 2 & TSS200 & 0.363 \\
\hline & 22 & RIMS1 & Regulating synaptic membrane exocytosis 1 & 4 & TSS200 & 0.316 \\
\hline
\end{tabular}

Abbreviations: Ctrl, control; GNAS, guanine nucleotide-binding protein Gs; Sb, spina bifida; TSS1500, the region of 1500 bp upstream of transcription start site of a gene; TSS200, the region of 200 bp upstream of transcription start site; ' 5 'UTR', 5' untranslated regions.

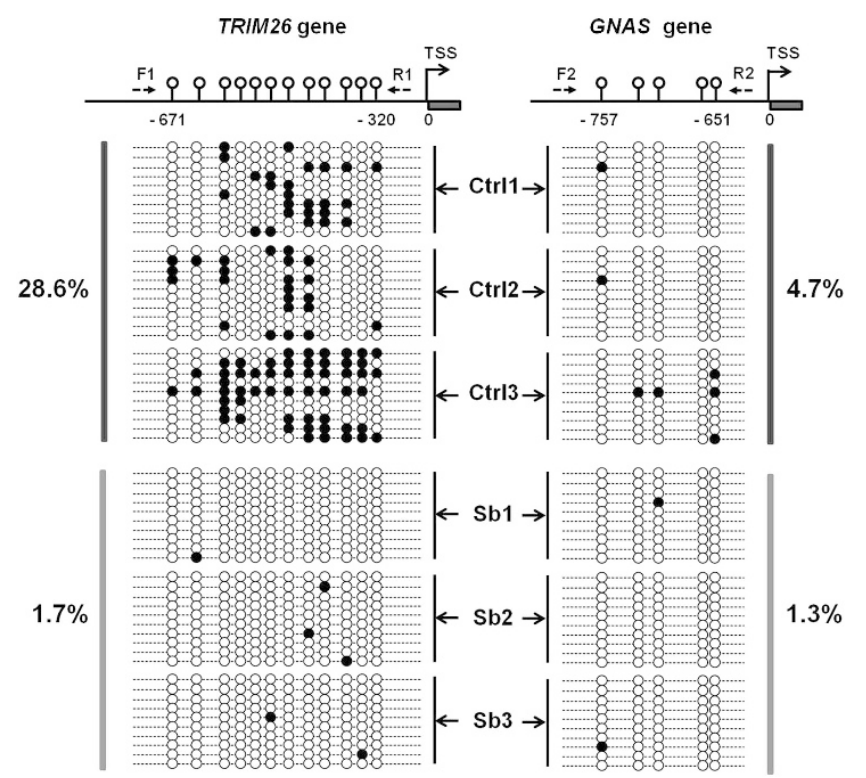

Figure 3 Bisulphite PCR sequencing in the TSS region of TRIM26 and GNAS. The sequencing regions are located into upstream of TSS (transcription start sites, 0). Circles along one column represented one CpG site (filled circles, methylated; open circles, unmethylated). Circles along one row represented one sequenced colony. The methylated site frequencies are labeled on both sides.

yet those effectors loci regulating neurotransmitter transport are in a state of hypermethylation $(\mathrm{Ctrl}<\mathrm{Sb})$. There are 20 sites located in TSS1500 of TRIM26 and covering $\sim 600 \mathrm{bp}$. The following experiments (Figures 3 and 4) provided evidence for epigenetic change causing high expression of TRIM26 protein in placentas of $\mathrm{Sb}$.
The protein expression of TRIM26 and GNAS is regulated by their DNA methylation statue in placentas

Next we identified the DNA methylation patterns of the TSS regions of TRIM26 and GNAS genes by bisulfite DNA sequencing. By designing a specific primer, DNA sodium bislufite modification, PCR amplification, TA-cloning and sequencing, we obtain the profiles of methylation sites in the regions to interest (Figure 3). From the result, the methylation frequencies in TSS1500 region of TRIM26 gene in control and $\mathrm{Sb}$ are 28.6 and 1.7\%, respectively. While those of GNAS are 4.7 and $1.3 \%$, respectively. This means the hypomethylation status in Sb placentas.

Based on the analysis, we tried to probe the change of the transcription and translation of the related genes in Sb. Because of the technical difficulties associated with isolating high-quality mRNA from placental samples, we determined the target protein levels in the placenta using western blotting. The results (Figure 4a) showed that the protein level of TRIM26 and GNAS were dramatically higher in Sb placenta than those in the normal controls, suggesting that a higher level of protein expression in the $\mathrm{Sb}$ group were linked to a significantly decreased level of DNA methylation of the target gene.

To test whether or not the protein levels of these genes are directly regulated by the DNA methylation level, we treated cell lines using 5-aza-2'-deoxycytidine, a DNA methytransferase inhibitor, and then tested the protein levels of GNAS and TRIM26. Interestingly we found that the protein expressions of TRIM26 and GNAS were dramatically elevated in $\mathrm{CHO}$ cells (a hamster ovary cell line) treated using 5-aza-2'-deoxycytidine (Figure 4b). Surprisingly in SW480 cells, a human colon cancer cell line, this drug treatment had no effect on these protein expressions, which may reflect the difference of methylation regulation in cancer cells from normal cells. Anyway 

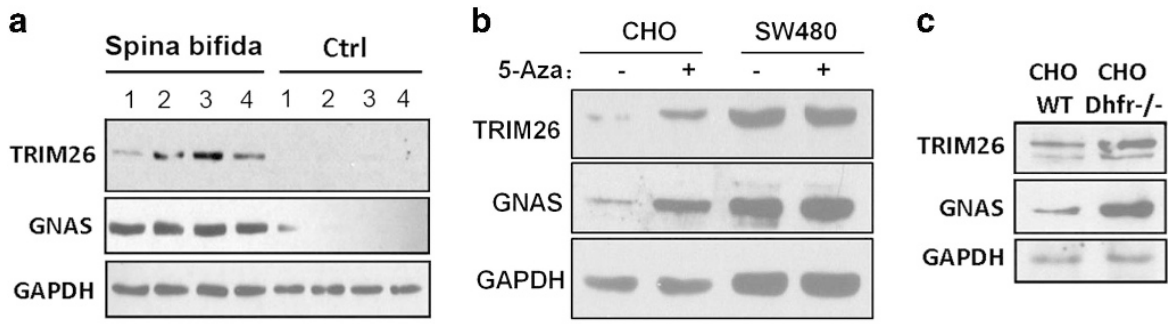

Figure 4 Protein expression levels of TRIM26 and GNAS in placenta. (a) Change in the protein expression in placenta of the fetus with spina bifida comparing with normal placenta. Total protein was extracted from fresh placenta samples ( $n=4$ each group). Western blot analysis was done using primary antibodies to TRIM26/GNAS and antibody to GAPDH as a control. (b) The protein levels of TRIM26 and GNAS were elevated after 5-aza-2'-deoxycytidine treatment in $\mathrm{CHO}$ cells. Cells were treated with 5-aza-2'-deoxycytidine (final concentration $10 \mu \mathrm{m}$ ) for $72 \mathrm{~h}$ and then harvested for western blot assay. (c) Change of TRIM26 and GNAS expression in normal $\mathrm{CHO}$ cells and $\mathrm{CHO}$ cells deficient of dihydrofolate reductase. Cells at the identical culture condition were harvested for western blot analysis.

the result in $\mathrm{CHO}$ cells showed that the protein levels of TRIM26 and GNAS are regulated by the DNA methylation level.

Next we tried to use a $\mathrm{CHO}$ cell line lack of dihydrofolate reductase to test the expression of these genes. Dihydrofolate reductase is a Key enzyme in folate metabolism and converts dihydrofolate into tetrahydrofolate, a methyl group shuttle. We found that in cells deficiency of dihydrofolate reductase the protein level of TRIM26 and GNAS is higher than that in normal cells (Figure 4c). The results suggested that deficiency in folate metabolism is probably associated with the protein level of these genes. Combining the above data we speculated that the expression of some key genes (for example, TRIM26) was changed in placentas of Sb because of the lack of folate intake, which contributes to the development of NTDs.

\section{DISCUSSION}

As an intermediary agent, the placenta transforms the information into its own genetic changes and directly passes on them to its fetus by accommodating embryonic metabolism and forming organs. Our results show for the first time that alteration of DNA methylation in placenta is closely associated with the pathological phenotype of fetal $\mathrm{Sb}$. Specially in Sb the number of hypomethylation sites is far more than that of hypermethylation sites, which suggests that a large number of genes are activated, transcribed and even translated in $\mathrm{Sb}$ placenta. Our exploration illuminated that DNA methylation in placenta affects its gene expression and thereby result in the deformity of fetal development.

To address whether or not the observed change in DNA methylation is placenta-specific, we carefully examined the available microarray data generated in Sb-affected fetuses in our laboratory. We noticed that, for the majority of genes that were also cross-examined in the Sb-affected fetuses, the mRNA levels in the Sb-affected fetuses showed no significant correlation with that of DNA methylation in the placenta (data not shown). Specifically, the mRNA levels for TRIM26 and GNAS in the Sb-affected fetuses showed no significant difference between $\mathrm{Sb}$ and control fetuses. This is a strong indication that the change of DNA methylation pattern in placenta is placenta-specific.

TRIM26 is a member of the tripartite motif family. The TRIM motif includes three zinc-binding domains (a RING, a B-box type 1 and a B-box type 2), and a coiled-coil region. Although the function of the protein is unclear, the RING domain suggests that the protein may have DNA-binding activity and metal ion binding. ${ }^{10}$ TRIM26 is a schizophrenia candidate gene by expression QTL analysis of top loci from GWAS meta-analysis. ${ }^{11}$ TRIM26 is differentially expressed in an independent set of schizophrenia cases and controls $(n=202)$.
Their work suggested that TRIM26 may involve in regulation of neural system function, which is consistent with our results.

Additionally GNAS is crucial signaling molecules mediating a number of cell signaling pathways. Our work suggested that its protein expression is regulated by DNA methylation and by this way their functions are related to NTDs. GNAS is an imprinted gene. Differentially methylated regions within GNAS are probably responsible for tissue-specific GNAS imprinting. ${ }^{12}$ Mutations on the paternal GNAS allele could be associated with intrauterine growth retardation and thus small size for gestational age, ${ }^{13}$ which indicated that GNAS transcript controls growth and fetal development by affecting placental functions. Our finding about GNAS gene provided a new evidence for GNAS vital function in fetal organ formation.

In addition in Table 2, we list our finding that methylation differential genes are classified into five categories: neurological system development and process; cell development and differentiation; transcription regulation; cell signaling and movement; and biosynthetic and metabolic process. Through further analysis we found the first kind (neurological development) covers the others which present the specific functions of these genes in cells. So, next we only presented the DNA methylation information of the genes related to neurological development in Table 3. However we still need explore and verify the relationship of their methylation status and expression level and try to dig more key genes associated with NTD phenotype. In these genes, there are many genes which are transcription factors involving in neurogenesis regulation, for example, $L B X 1, V A X 1$ and $L M X 1 A$. FEZF1 could regulate neuron migration which is crucial for the formation of fetal neural tube. Other more, GDNF, BDNF and NMUR1 are some neural signaling molecules mediating neurodevelopmental signal transmission. Illuminating their gene regulation styles will help explain the mechanism of the effect of placenta on fetal neurodevelopment.

In future work we will increase the number of tested differential genes for obtaining more comprehensive information of protein expression and regulating network of Sb. In addition, we will focus on how TRIM26 and GNAS genes regulate the occurrence of NTDs. We will analyze their DNA methylation status in $\mathrm{CHO}$ cells and build up the direct correction to DNA methylation modification and their protein levels with folate metabolism. More we can set up their mouse models serving to resolve the molecular mechanism of neural tube development.

In conclusion our result showed that DNA methylation patterns of placenta are significantly associated with fetal $\mathrm{Sb}$, enlightened the placenta's vital role in organ development and provided a new insight in researching the molecular mechanism of NTDs. 


\section{CONFLICT OF INTEREST}

The authors declare no conflict of interest.

\section{ACKNOWLEDGEMENTS}

We thank Quanbin Xu for critical readings of the manuscript. We appreciate Xiujie Wang and Tong Chen providing the suggestions of data treatment and statistics. This work was supported by grants from the National Natural Science Foundation of China to Xiaojuan Zhang (No. 31101013), the National Basic Research Program of China (No. 2007CB511901 and No. 2014CB138503) and the State Key Laboratory for Molecular Developmental Biology (No. 2013-MDB-ZD-04).

1 Greene, N. D., Stanier, P. \& Moore, G. E. The emerging role of epigenetic mechanisms in the etiology of neural tube defects. Epigenetics 6, 875-883 (2011).

2 Botto, L. D., Moore, C. A., Khoury, M. J. \& Erickson, J. D. Neural-tube defects. N. Engl. J. Med. 341, 1509-1519 (1999)

3 Gu, X., Lin, L., Zheng, X., Zhang, T., Song, X., Wang, J. et al. High prevalence of NTDs in Shanxi Province: a combined epidemiological approach. Birth Defects Res. A Clin. Mol. Teratol. 79, 702-707 (2007)

4 Maccani, M. A. \& Marsit, C. J. Epigenetics in the placenta. Am. J. Reprod. Immunol. 62, 78-89 (2009).
5 Larque, E., Ruiz-Palacios, M. \& Koletzko, B. Placental regulation of fetal nutrient supply. Curr. Opin. Clin. Nutr. Metab. Care 16, 292-297 (2013).

6 Banister, C. E., Koestler, D. C., Maccani, M. A., Padbury, J. F., Houseman, E. A. \& Marsit, C. J. Infant growth restriction is associated with distinct patterns of DNA methylation in human placentas. Epigenetics 6, 920-927 (2011).

7 Suter, M., Ma, J., Harris, A., Patterson, L., Brown, K. A., Shope, C. et al. Maternal tobacco use modestly alters correlated epigenome-wide placental DNA methylation and gene expression. Epigenetics 6, 1284-1294 (2011).

8 Sandoval, J., Heyn, H., Moran, S., Serra-Musach, J., Pujana, M. A., Bibikova, M. et al. Validation of a DNA methylation microarray for $450,000 \mathrm{CpG}$ sites in the human genome. Epigenetics 6, 692-702 (2011).

9 Xia, K., Xue, H., Dong, D., Zhu, S., Wang, J., Zhang, Q. et al. Identification of the proliferation/differentiation switch in the cellular network of multicellular organisms. PLoS Comput. Biol. 2, e145 (2006).

10 Reymond, A., Meroni, G., Fantozzi, A., Merla, G., Cairo, S., Luzi, L. et al. The tripartite motif family identifies cell compartments. EMBO J. 20, 2140-2151 (2001).

11 de Jong, S., van Eijk, K. R., Zeegers, D. W., Strengman, E., Janson, E., Veldink, J. H. et al. Expression QTL analysis of top loci from GWAS meta-analysis highlights additional schizophrenia candidate genes. Eur. J. Hum. Genet. 20, 1004-1008 (2012).

12 Izzi, B., Van Geet, C. \& Freson, K. Recent advances in GNAS epigenetic research of pseudohypoparathyroidism. Curr. Mol. Med. 12, 566-573 (2012).

13 Richard, N., Molin, A., Coudray, N., Rault-Guillaume, P., Jüppner, H. \& Kottler, M. L. Paternal GNAS mutations lead to severe intrauterine growth retardation (IUGR) and provide evidence for a role of XLalphas in fetal development. J. Clin. Endocrinol. Metab. 98, E1549-E1556 (2013).

Supplementary Information accompanies the paper on Journal of Human Genetics website (http://www.nature.com/jhg) 